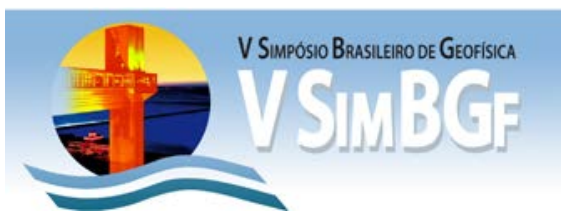

\title{
Inversão conjunta SEVITDEM aplicada em estudos hidrogeológicos na região de Urupês-SP, bacia do Paraná: Resultados preliminares
}

*David Nakamura Leite \& Jorge Luís Porsani, Instituto de Astronomia, Geofísica e Ciências Atmosféricas (IAG/USP).

Copyright 2012, SBGf - Sociedade Brasileira de Geofísica

Este texto foi preparado para a apresentação no $V$ Simpósio Brasileiro de Geofísica, Salvador, 27 a 29 de novembro de 2012. Seu conteúdo foi revisado pelo Comitê Técnico do V SimBGf, mas não necessariamente representa a opinião da SBGf ou de seus associados. É proibida a reprodução total ou parcial deste material para propósitos comerciais sem prévia autorização da SBGf.

\begin{abstract}
The use of geophysical methods in groundwater exploration has become an important tool to assess the best points of exploitation. This paper show preliminary results of joint inversion of SEV and TDEM for Urupês region, Paraná basin. The objectives of this research were to map the Adamantina Formation (sedimentary aquifer) and to locate fractures zones in the basalt layers of the Serra Geral Formation. The results indicate the tick of the Adamantina Formation and the fracture zones in the basalt layer of the Serra Geral Formation.
\end{abstract}

\section{Introdução}

No Estado de São Paulo, 462 municípios são abastecidos por águas subterrâneas, abrangendo uma população de 5,5 milhões de pessoas que dependem de alguma forma desse recurso (Iritani \& Ezaki, 2009). Nesse contexto, insere-se a região de Urupês que está localizada na porção noroeste do Estado de São Paulo, a aproximadamente $450 \mathrm{~km}$ da capital (Figura 1).

Nos últimos anos, o município de Urupês está tendo problemas com a falta de abastecimento de água no período do verão. O aumento do consumo e a diminuição de recarga de água são os principais agravantes. Uma rápida solução é a locação de novos poços de exploração de água subterrânea para equilibrar o abastecimento local. Para isso, a geofísica através dos métodos elétricos e eletromagnéticos pode oferecer, de maneira efetiva, os locais mais favoráveis para a perfuração de novos poços.

O uso integrado de métodos distintos e o tratamento dos dados por meio da inversão conjunta de diferentes métodos permitem reduzir a ambiguidade no processo interpretativo. A inversão conjunta de dados elétricos (SEV) e eletromagnéticos no domínio do tempo (TDEM) ressalta as vantagens de cada método. As SEV's definem melhor as camadas resistivas, ao passo que, as sondagens TDEM definem melhor as camadas condutivas (Schmutz et al., 2000). Assim, as ambiguidades presentes nos dados são reduzidas e a interpretação torna-se mais confiável.

Geologicamente, a região de Urupês encontra-se assentada na Bacia do Paraná sobre as formações (da base para o topo): Botucatu, Serra Geral e Adamantina. A Formação Botucatu é constituída por arenitos de granulação fina a grossa, sendo caracterizada por estratificações cruzadas de grande porte que indicam uma sedimentação em ambiente eólico-desértico. Sobre a Formação Botucatu encontra-se a Formação Serra Geral, sendo caracterizada essencialmente por basaltos originados do derramamento basáltico há aproximadamente $130 \mathrm{Ma}$. Segundo dados de poços obtidos no DAEE de Araraquara, a espessura do basalto pode variar de $400 \mathrm{~m}$ a $800 \mathrm{~m}$ na região.

Sobre a Formação Serra Geral encontra-se a Formação Adamantina que se caracteriza pela presença de arenitos de granulação fina a muito fina com espessura entre $\sim 70 \mathrm{~m}$ e $100 \mathrm{~m}$. A Formação Adamantina abriga o aquífero Bauru que, por ser de fácil acesso e boa qualidade, é amplamente explorado na região de Urupês. As vazões sustentáveis dos poços sobre esse aquífero, segundo Iritani \& Ezaki (2009) é de aproximadamente $10 \mathrm{~m}^{3} / \mathrm{h}$ na região. Essa vazão não é suficiente para abastecer a cidade, por isso a necessidade de se construir um poço que atinja o aquífero Serra Geral, com vazões entre $23 \mathrm{~m}^{3} / \mathrm{h}$ e $190 \mathrm{~m}^{3} / \mathrm{h}$ (Iritani \& Ezaki, 2009) ou o aquífero Botucatu, que pode apresentar uma vazão entre $360 \mathrm{~m}^{3} / \mathrm{h}$ e $1000 \mathrm{~m}^{3} / \mathrm{h}$ (Gualdi, 1999).

\section{Metodologia}

Uma nova abordagem metodológica de inversão conjunta de dados de SEV e sondagens TDEM é empregada neste trabalho, visando mapear a estratigrafia geoelétrica na região de Urupês-SP em apoio à perfuração de poços tubulares para abastecimento de água para a comunidade local.

Os dois métodos possuem vantagens e desvantagens, e quando são usados em conjunto, se complementam. O método TDEM é amplamente utilizado em estudos hidrogeológicos (Christiansen et al., 2006; Nielsen et al., 2006; Porsani et al., 2012a, 2012b) devido à capacidade de investigação em grandes profundidades. Além disso, este método destaca-se pela capacidade de definir melhor as estruturas condutivas. O método geoelétrico, também pode ser aplicado para pesquisas hidrogeológicas em grandes profundidades, mas detalha melhor as camadas mais superficiais e estruturas resistivas. Desta forma, a inversão conjunta proporciona uma melhor estimativa dos parâmetros como resistividade e espessura. Essas informações, analisadas em conjunto, reduzem as ambiguidades presentes nos dados e ajudam o geofísico definir melhor as estruturas geológicas em subsuperfície (Bortolozo, 2011).

Os dados foram adquiridos em duas campanhas de campo (2009 e 2011), totalizando 15 sondagens TDEM e 8 SEV's. Na primeira campanha, em 2009, foram adquiridas 6 sondagens TDEM. Ao passo que, na 
segunda campanha, adquiriu-se 9 sondagens TDEM e 8 SEV'S.

Cada sondagem TDEM foi obtida com o equipamento PROTEM-57-MK2, arranjo loop-central com geometria quadrada de $100 \mathrm{~m} \times 100 \mathrm{~m}$. Os dados foram adquiridos em 3 frequências: $30 \mathrm{~Hz}, 7,5 \mathrm{~Hz}$ e $3 \mathrm{~Hz}$ que investigam em diferentes níveis de profundidade. Em cada frequência foram obtidas 3 curvas num intervalo de tempo de 360s (120s para cada curva). Ao final do processo, tem se uma única curva com as três frequências citadas acima. Esse processo foi repetido para cada uma das 15 sondagens TDEM.

Os dados de sondagem elétrica vertical foram adquiridos com o equipamento SYSCAL-R2, eletrodos porosos, usando o arranjo Schlumberger. $O$ arranjo, com $A B / 2$ de $200 \mathrm{~m}$, foi posicionado de maneira que o centro fosse coincidente com o centro da sondagem TDEM.

Após o término das aquisições, os dados foram tratados e invertidos separadamente. O tratamento dos dados TDEM consistiu em eliminar os pontos espúrios que estavam espalhados em relação à tendência da curva de resistividade aparente. Todo o procedimento acima foi realizado usando o software IX1D (Interpex). O processo de inversão conjunta SEV/TDEM foi realizado no software "Curupira" (Bortolozo \& Porsani, 2012) que permite a inversão dos dados TDEM e SEV de maneira conjunta e separada. Este software permite realizar a correção do static-shift presente nas sondagens elétricas verticais através das sondagens TDEM's, por isso a necessidade de fazer aquisições com centros coincidentes. Mais informações a respeito do static-shift, bem como as formulações necessárias para sua correção podem ser encontradas no artigo de Meju (2005).

\section{Resultados}

A Figura 2 mostra o resultado da sondagem 14 (S14). Inicialmente, nota-se que o ajuste foi muito bom para a inversão conjunta, visto que, os erros de ajuste ficaram abaixo de $5 \%$. Por volta dos $2 \mathrm{~m}$ de profundidade, iniciase a Formação Adamantina (aquífero raso) que se estende até os $54 \mathrm{~m}$ de profundidade. Nota-se que o contato entre a Formação Adamantina e Serra Geral é bem marcante. Com base em informações de poços na região este contato ocorre em $\sim 60$ m de profundidade.
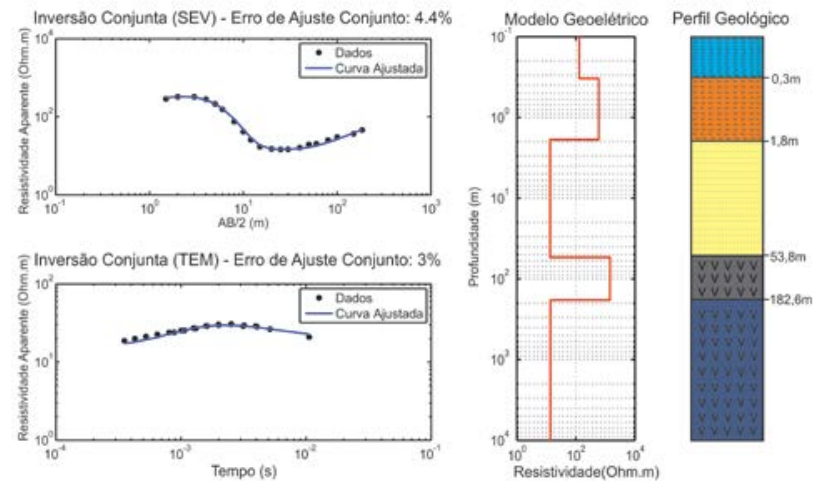

Figura 2. Inversão conjunta dos dados SEV e TDEM da S14. Modelo geoelétrico e perfil litológico interpretativo

A partir dos $54 \mathrm{~m}$ de profundidade tem-se uma camada muito resistiva (1400 ohm.m). Essa camada foi interpretada como basaltos da Formação Serra Geral e sua espessura é de aproximadamente 129m. Abaixo dessa camada, verifica-se a presença de uma zona condutiva. Inicialmente, esses resultados seriam interpretados como arenitos intertraps. Esses sedimentos (intertraps) teriam sido acomodados durante os períodos de interrupção do derramamento magmático. Por outro lado, a zona condutiva, abaixo do basalto, pode indicar fraturas preenchidas com água e/ou argilas.

A Figura 3 apresenta os resultados da sondagem 7 (S07), onde os erros de ajustes foram menores que $3 \%$. Os resultados apresentam um modelo de 6 camadas, sendo que as duas primeiras camadas correspondem ao solo úmido e seco. Esperava-se um ajuste com apenas uma única camada para o solo. No entanto, durante o processo de inversão, verificou-se que a qualidade do ajuste seria inferior com um modelo de 5 camadas.

A terceira camada inicia-se a $6 \mathrm{~m}$ de profundidade e sua espessura é de aproximadamente $22 \mathrm{~m}$. Com uma resistividade intermediária, essa camada pode ser interpretada como a zona de capilaridade. Pois representa a transição do solo insaturado para os arenitos saturados da Formação Adamantina. Os arenitos saturados representam a quarta camada geoelétrica, com uma espessura de $52 \mathrm{~m}$, essas rochas constituem a principal rocha reservatório de água da Formação Adamantina (Iritani e Ezaki, 2009).

Abaixo da Formação Adamantina, encontra-se uma camada mais resistiva que é interpretada como basalto da Formação Serra Geral. Sua espessura é de 190m e, a partir dos $270 \mathrm{~m}$ de profundidade, há uma queda nos valores de resistividade podendo estar relacionada à presença de fraturas no basalto com água e/ou argila. 


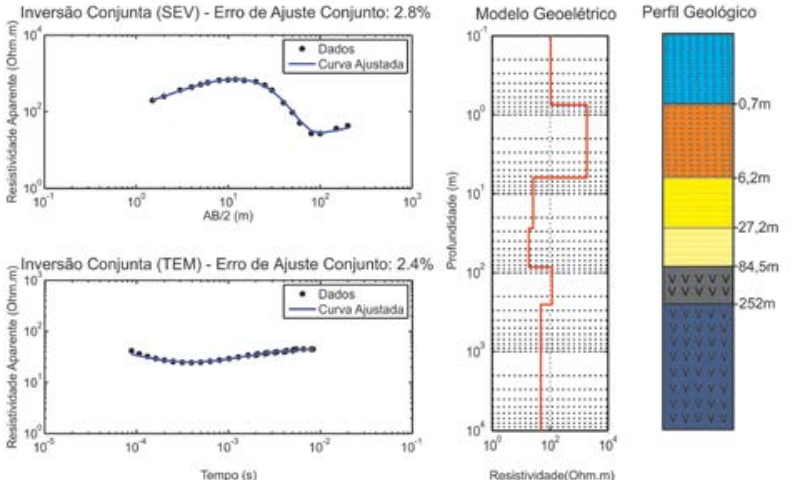

Figura 3. Inversão conjunta dos dados SEV e TDEM da S07. Modelo geoelétrico e perfil litológico interpretativo.

\section{Conclusões}

Com base nos modelos geoelétricos obtidos nas 15 sondagens TDEM, 8 SEV's e informações de poços foi possível identificar duas importantes feições geológicas. O contato entre as Formações Adamantina e Serra Geral e fraturas localizadas nos basaltos. O contato entre as formações indica o limite do Aquífero Bauru que, na região, varia de $60 \mathrm{~m}$ a $80 \mathrm{~m}$ de profundidade. Essa informação poderá auxiliar na implementação de novos poços, à medida que, o conhecimento da espessura do aquífero é parâmetro para a estimativa de vazão dos poços.

A presença de fraturas no basalto localizadas nas sondagens 7 e 8 , a $270 \mathrm{~m}$ e $184 \mathrm{~m}$ de profundidade respectivamente, indicam possíveis locais de perfuração. A perfuração de um poço no Aquífero Serra Geral poderia implementar uma vazão média de $23 \mathrm{~m}^{3} / \mathrm{h}$. No entanto, há registros na literatura de poços com vazões variando de zero a $100 \mathrm{~m}^{3} / \mathrm{h}$ (Iritani \& Ezaki, 2009). Desta forma, recomenda-se que o poço seja perfurado nas imediações da sondagem 8 , pois, a espessura do basalto é menor e, por isso, os custos de perfuração seriam reduzidos. Além disso, o fato dessa sondagem encontrarse dentro do perímetro urbano facilitaria a distribuição de água na cidade.

\section{Agradecimentos}

Ao CNPq pela bolsa de Mestrado. Ao IAG pela infraestrutura e oportunidade para realizar esta pesquisa. Ao José Luiz, geólogo do DAEE de Araraquara, por fornecer dados de poços da região de estudos. Aos amigos Cassiano, Divanir, Emerson, Ernande, Marcelo e Thiago por colaborarem nas várias etapas desse trabalho.

\section{Referências}

Bortolozo, C.A.; 2011. Inversão Conjunta 1D de Dados de SEV e TDEM: Aplicações em Hidrogeologia - SP. Tese de Mestrado, IAG/USP.

Bortolozo, C.A.; Porsani, J.L., 2012. Curupira v1.0. Software de inversão conjunta 1D de sondagens SEV/TDEM. Registro de Software no. 12988-1. Revista da Propriedade Industrial no. 2165, item 090, p.145.

Christiansen, A.V., Auken, E., Sørensen, K., 2006, The transient electromagnetic method, in R. Kirsch ed., Groundwater geophysics a tool for hydrogeology, Cap.6, 179-225pp.

Gualdi, O. J., 1999. Caracterização do Sistema Aqüífero Guarani. In: Seminário Gestão do Aqüífero Guarani, Ribeirão Preto. Resumos, São Paulo: SMA. 2 p.

Iritani, M.A.; Ezaki, S. As águas subterrâneas do Estado de São Paulo. 2 ed. São Paulo: Secretaria de Estado do Meio Ambiente - SMA, 2009. 104p.

Meju, M.A., 2005. Simple relative space-time scaling of electrical and electromagnetic depth sounding arrays: implication for electrical static shift removal and joint DCTEM data inversion with the most squares-criterion: Geophysical Prospecting, 53, 1-17.

Porsani, J.L., Bortolozo, C.A., Almeida, E.R., Santos Sobrinho, E. N., Santos, T.G., 2012a. TDEM survey in urban environmental for hydrogeological study at USP campus in São Paulo city, Brazil. Journal of Applied Geophysics 76, 102-108.

Porsani, J.L., Almeida, E.R., Bortolozo, C.A., Monteiro Santos, F.A., 2012b. TDEM survey in an area of seismicity induced by water wells in Paraná sedimentary basin, Northern São Paulo State, Brazil. Journal of Applied Geophysics 82, 75-83.

Schmutz, M., Albouy, Y., Guerin, R., Maquaire, O., Vassal, J., Schott, J.J., Descloitres, M., 2000. Joint electrical and time domain electromagnetism (TDEM) data inversion applied to the Super Sauze earthflow (France): Surveys in Geophysics, 21, 371-390. 

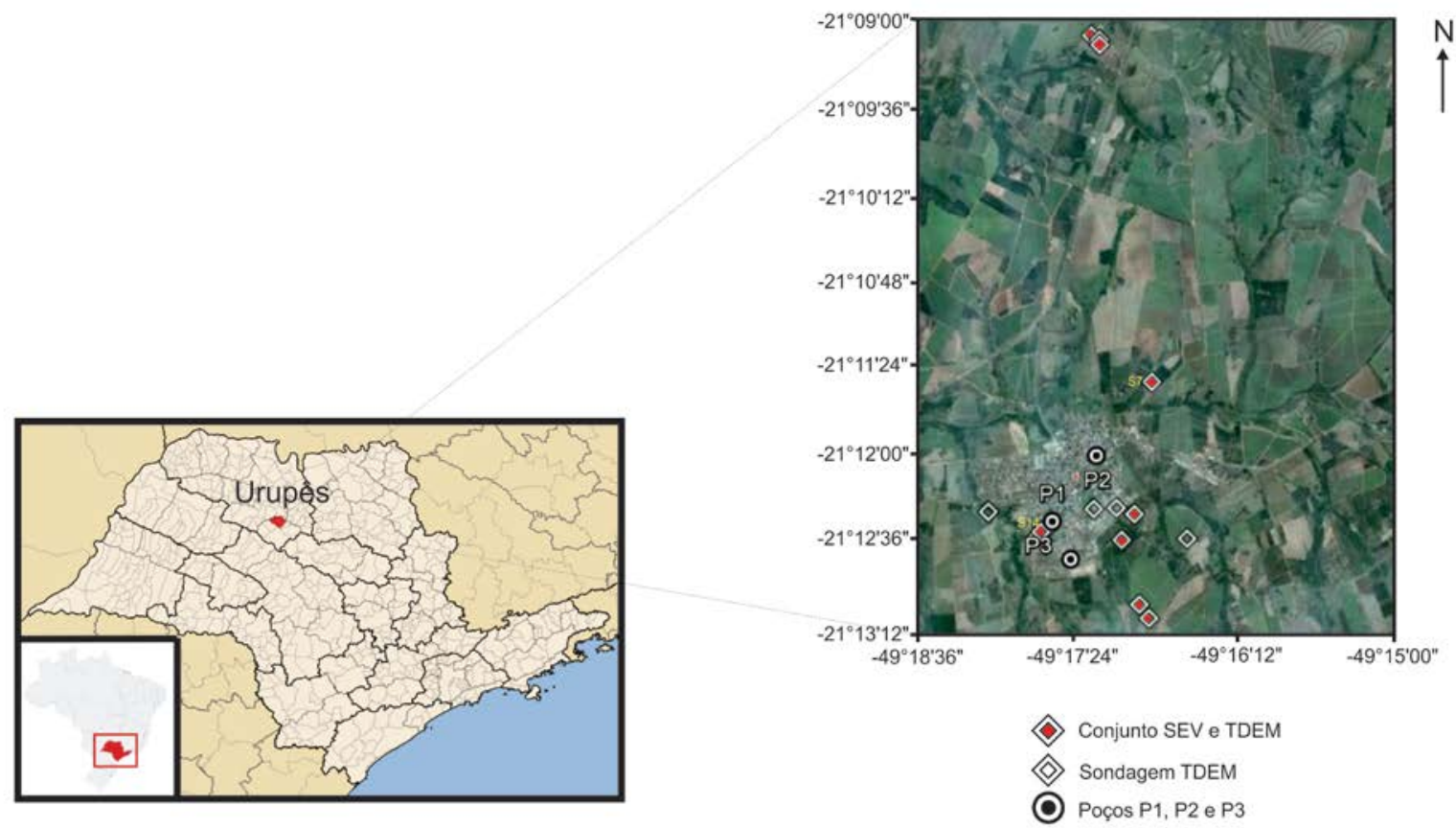

Figura 1. Localização do município de Urupês, Estado de São Paulo, as sondagens SEV e TDEM, e os poços de exploração de água subterrânea. 